\title{
PRÉ-TRATAMENTO POR DEPOSIÇÃO ELETROASSISTIDA DE VINILTRIETOXISSILANO (VTES) PARA A PINTURA DE ALUMÍNIO
}

\author{
Vitor Bonamigo Moreira ' \\ Jane Zoppas Ferreira' \\ Álvaro Meneguzzi '
}

\section{Resumo}

A técnica de deposição eletroassistida pode conduzir à formação de filmes de organossilanos mais contínuos pelo método de sol-gel. A técnica baseia-se na promoção da eletrólise da água para aumentar o pH na região catódica, o que acelera as reações de condensação do precursor organossilano no cátodo e resulta em filmes reticulados que cobrem o substrato mais homogeneamente em comparação à técnica de imersão simples. Neste estudo utilizou-se viniltrietoxissilano (VTES) como precursor no método de sol-gel para a produção de revestimentos pela técnica de deposição eletroassistida como pré-tratamento para a pintura de alumínio com verniz poliuretânico. Tais revestimentos foram comparados à cromatização e à técnica de imersão simples por ensaio de corrosão acelerada em névoa salina e medida de aderência de revestimento orgânico e foram caracterizados morfologicamente por MEV e EDS. Os resultados indicam que a deposição eletroassistida promove a formação de filmes protetores mais homogêneos e propicia o aumento da aderência do verniz utilizado.

Palavras-chave: Silano; Deposição eletroassistida; Pré-tratamento; Alumínio.

\section{PRETREATMENT FOR PAINTING ALUMINUM BY ELECTRO-ASSISTED DEPOSITION OF VINYLTRIETHOXYSILANE (VTES)}

\begin{abstract}
The electro-assisted deposition technique may lead to more continuous silane films by the sol-gel method. This technique is based on promoting water electrolysis to increase the $\mathrm{pH}$ on the cathodic region, which accelerates the silane condensation reactions on the cathode and results in crosslinked films that cover the substrate more homogeneously in comparison to the dip-coating technique. In this study, vinyltriethoxysilane (VTES) was used as a precursor in the sol-gel method for the production of coatings by electro-assisted deposition as a pretreatment for painting aluminum with polyurethane varnish. These coatings were compared to chromate and dip-coating pretreatments by salt spray test, paint adhesion measurement and were morphologically analyzed by SEM and EDS. The results indicate that the electro-assisted deposition technique leads to the formation of more homogeneous protective films and improves the adherence of the tested varnish.
\end{abstract}

Keywords: Silane; Electro-assisted deposition; Pretreatment; Aluminum.

\section{INTRODUÇÃO}

A deposição eletroassistida de filmes a partir de precursores hidrolisados em sol-gel é uma alternativa para a produção de revestimentos com menos descontinuidades e com melhores propriedades de proteção que a técnica convencional de imersão simples [I]. O fundamento no qual a técnica de deposição eletroassistida se embasa é o de que os organossilanos apresentam reações concorrentes de hidrólise e condensação [2], sendo que, de modo geral, as reações de hidrólise são favorecidas em pH ácido enquanto que um $\mathrm{pH}$ alcalino aumenta a taxa das reações de condensação [3]. Deste modo, através da aplicação de potencial em uma célula eletroquímica onde a solução hidroalcoólica do precursor é o eletrólito, promove-se a eletrólise da água [4], composta pelas seguintes reações:

$$
\text { Reação catódica: } 2 \mathrm{H}_{2} \mathrm{O}+2 \mathrm{e}^{-} \rightarrow \mathrm{H}_{2}+2 \mathrm{OH}^{-}
$$

'Laboratório de Corrosão, Proteção e Reciclagem de Materiais, Universidade Federal do Rio Grande do Sul - UFRGS, Porto Alegre, RS, Brasil.

E-mail: vitor.bonamigo@hotmail.com

2176-1523 (C) 2017 Associação Brasileira de Metalurgia, Materiais e Mineração. Publicado pela ABM. Este é um artigo de acesso aberto distribuído sob os termos da licença Creative Commons CC BY-NC-ND (Attribution-NonCommercial-NoDerivs) - https:// creativecommons.org/licenses/by-nc-nd/4.0\%. 
Reação anódica: $2 \mathrm{H}_{2} \mathrm{O} \rightarrow 4 \mathrm{H}^{+}+\mathrm{O}_{2}+4 \mathrm{e}^{-}$

Como a reação catódica tem como produto o íon hidroxila, há um aumento localizado do $\mathrm{pH}$, aumentando a formação das pontes de hidrogênio entre as espécies hidrolisadas do precursor na superfície do cátodo, resultando em uma cobertura mais homogênea da superfície por filmes de organossilano mais espessos [4]. Adicionalmente, a técnica possibilita a incorporação de outros elementos no filme de organossilano sem a necessidade de alterações no procedimento [5,6], expandindo as possibilidades do seu uso.

A aderência de revestimentos orgânicos ao alumínio é prejudicada em virtude da inércia química da sua camada natural de óxido, logo há a necessidade do uso de pré-tratamentos cuja função é promover alterações superficiais que resultem em maior aderência em função de ancoragem química ou mecânica [7]. Dentre estes pré-tratamentos, o de uso mais difundido é a cromatização, que promove aderência de tintas através de ligações secundárias [8] e fornece proteção por barreira contra a corrosão com propriedades de autocura [9].

O uso do processo de cromatização, aceito por várias décadas, tem enfrentado limitações em função do seu potencial poluidor, uma vez que gera efluentes ricos em cromatos, que apresentam risco em caso de disposição ou tratamento inadequado, além de representarem um risco aos trabalhadores envolvidos no processo [10]. Dentre as alternativas em desenvolvimento para a cromatização como pré-tratamento de ligas de alumínio estão os revestimentos à base de organossilanos, cuja escolha adequada pode promover não somente a proteção por barreira contra a corrosão [2], mas também a aderência de tintas e vernizes em função de afinidade química entre o grupamento organofuncional do pré-tratamento e o revestimento orgânico [ | | - |3].

O uso de organossilanos não-funcionalizados para a produção de pré-tratamentos para a pintura de aço já foi estudado por Wang et al. [14], porém, em virtude da falta de grupamentos funcionais que pudessem promover a aderência de tinta, a técnica de deposição eletroassistida foi empregada para a obtenção de filmes de $\mathrm{SiO}_{2}$, cuja função era favorecer a aderência mecânica do revestimento orgânico. A este revestimento, em trabalho posterior [15], foi acrescentada outra camada utilizando-se um organossilano com grupamento funcional epóxi com o objetivo de propiciar também a aderência química da tinta, mostrando o papel fundamental da afinidade química entre o organossilano e a camada final de tinta para a obtenção de aderência satisfatória do revestimento orgânico.

Deste modo, o presente trabalho tem o objetivo de produzir filmes à base de precursor organossilano a partir da técnica de deposição eletroassistida empregando-os como pré-tratamento para a pintura de um substrato de alumínio, comparando o desempenho desses revestimentos com pré-tratamentos produzidos por imersão simples e também com a cromatização.

Neste trabalho foi utilizado o precursor viniltrietoxissilano (VTES), cuja proteção contra a corrosão já foi observada anteriormente em aço inoxidável [16], seguido da aplicação de revestimento poliuretânico, de modo a reproduzir um sistema de pré-tratamento + revestimento orgânico bem-sucedido anteriormente [17], porém visando o aprimoramento do sistema a partir do uso da deposição eletroassistida.

A comparação entre os sistemas de revestimentos foi realizada através de ensaio de corrosão acelerada em névoa salina, medida de aderência de revestimento orgânico por tração, microscopia eletrônica de varredura (MEV) e espectroscopia de energia dispersiva (EDS). Adicionalmente, foi realizada uma voltametria na célula eletroquímica de deposição eletroassistida.

\section{MATERIAIS E MÉTODOS}

\section{I Preparo das Amostras}

Foram utilizadas como substrato placas de alumínio comercialmente puro AA I 100 de dimensões $100 \mathrm{~mm} \times 50 \mathrm{~mm} \times 0,5 \mathrm{~mm}$. Estas peças foram desengraxadas com pano embebido em acetona (Acetona P. A. - Neon Comercial Ltda.), em seguida foram imersas em desengraxante alcalino (Saloclean $667 \mathrm{~N}$ - Klintex Insumos Industriais Ltda.) a $70^{\circ} \mathrm{C}$ durante 5 minutos. Após a imersão em desengraxante as placas foram enxaguadas com água destilada, sendo posteriormente secadas com jato de ar quente.

A solução utilizada para a produção dos revestimentos por sol-gel foi composta, em proporções volumétricas, por $6 \%$ de VTES (Aldrich Chemistry 97\%), 47\% de álcool etílico (Synth ${ }^{\circledR} 95 \%$ P. A.) e 47\% de água destilada deionizada. O pH da solução foi regulado a 4 com a adição de ácido acético glacial $\left(\right.$ Synth $\left.^{\circledR}\right)$. Após o preparo, a solução foi mantida sob agitação por 24 horas para a ocorrência das reações de hidrólise do precursor.

A produção dos revestimentos de VTES por imersão simples foi realizada em um Elevador de Discos MA 765 (Marconi Equipamentos para Laboratórios Ltda.), com tempo de imersão de 5 minutos e retirada à taxa de $210 \mathrm{~mm} / \mathrm{min}$. Para as amostras produzidas por deposição eletroassistida foi utilizada uma célula de três eletrodos, sendo um eletrodo de referência de $\mathrm{Ag} \mid \mathrm{AgCl}$, contra eletrodos de aço inoxidável AISI 316 e a placa a ser revestida como o eletrodo de trabalho, ligada a um potenciostato (Autolab PGSTAT 302, software de operação Nova I.I I). Após a montagem das células, foi medido o potencial de circuito aberto (OCP) dos sistemas durante 10 segundos. Findada esta medida, os sobrepotenciais de deposição foram aplicados às células, com relação aos seus respectivos valores de OCP.

Tanto as amostras revestidas por imersão quanto por deposição eletroassistida passaram por secagem em estufa a $50^{\circ} \mathrm{C}$ por 10 minutos para a remoção do excesso de líquidos presente nos filmes, após a qual a cura dos revestimentos de VTES foi realizada durante 60 minutos a $125^{\circ} \mathrm{C}$.

As peças a serem cromatizadas passaram do desengraxe à imersão em solução cromatizante hexavalente (Cromatizante Hexavalente 674B - SurTec do Brasil Ltda.) por 5 minutos, seguida de retirada à taxa de $210 \mathrm{~mm} / \mathrm{min}$, 
com o equipamento elevador de discos anteriormente citado. Este procedimento foi sucedido com secagem por jato de ar quente.

As amostras sem pré-tratamento seguiram da etapa de desengraxe diretamente à etapa de pintura.

A aplicação do revestimento orgânico (Verniz Poliuretânico Brilhante - Majestic Vernizes) foi realizada para todas as amostras por imersão simples em equipamento Elevador de Discos MA 765 durante 5 segundos, seguida de retirada a $210 \mathrm{~mm} / \mathrm{min}$. Após a pintura, as amostras passaram por um período de secagem em exposição ao ar atmosférico à temperatura ambiente por 24 horas.

Ao término deste procedimento, as amostras receberam as seguintes denominações: Branco, para as amostras sem pré-tratamento; Cromatizado, para amostras cromatizadas; Imersão para as amostras que passaram somente pela deposição via imersão simples; $-0,4 \mathrm{~V},-0,8 \vee$ e - I,2 $\vee$ para as amostras produzidas por deposição eletroassistida nesses respectivos sobrepotenciais.

\subsection{Ensaios}

Foi realizada uma voltametria na célula de deposição eletroassistida, simulando-se a montagem de deposição em potenciostato (Autolab PGSTAT 302, software de operação Nova I. I I), com varredura de - I,5 $\mathrm{V}$ a $+0,5 \mathrm{~V}$ com relação ao OCP à taxa de $0,01 \mathrm{~V} / \mathrm{s}$.

O ensaio de corrosão acelerada em névoa salina foi conduzido conforme a norma ABNT NBR 8094, com avaliação periódica do grau de corrosão. Os graus de corrosão vão de 0 (zero) a 5 , sendo 0 a ausência de corrosão e 5 a corrosão generalizada da peça.

A medida de aderência do revestimento orgânico com equipamento portátil (DeFelsko PosiTest AT-A) foi realizada de acordo com a norma ASTM D454 I-09. Foram empregadas dollies de $20 \mathrm{~mm}$ de diâmetro e a taxa de tração utilizada foi de 0,2 MPa/s.

As análises de MEV e EDS (Zeiss EVO MA I0) foram realizadas com o intuito de avaliar a integridade do filme formado. Para tanto, foram obtidas micrografias de elétrons retroespalhados com magnificação de 52x e, nas mesmas regiões, foi realizado o mapeamento do silício presente nas amostras, que tem como resultado imagens de fundo preto onde são marcados pontos em branco e em tons de cinza referentes à presença de silício. Com o uso de um software de análise de imagem (Image]) foi calculada a área proporcional contendo silício nestes mapas.

\section{RESULTADOS E DISCUSSÃO}

\section{I Voltametria}

O ensaio de voltametria realizado na célula de deposição eletroassistida simulando a montagem utilizada para as deposições de VTES resultou no voltamograma mostrado na Figura I.

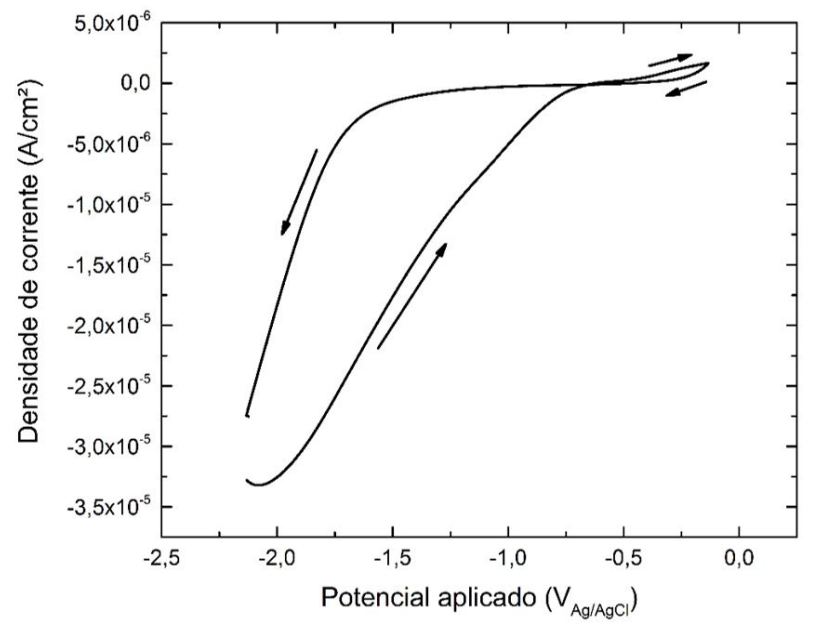

Figura I. Voltamograma conduzido na célula de deposição eletroassistida com substrato de alumínio AA I 100 em sol-gel de VTES a partir da varredura de potenciais entre $-I, 5 \vee$ e $+0,5 \vee$ a partir do OCP.

O OCP medido para a célula foi de $-0,62 \mathrm{~V}_{\mathrm{Ag} \mid \mathrm{Ag}_{\mathrm{g} C l}}, \mathrm{e}$ não é evidente a ocorrência de nenhuma reação de redução ou oxidação nas espécies presentes na solução, indicando que possivelmente a eletrólise da água seja a única reação ocorrendo ao longo do ciclo.

Trabalhos anteriores de outros autores [18-20] indicam que, para diferentes organossilanos, o sobrepotencial $-0,8 \mathrm{~V}_{\text {ocP, }}$ resulta na formação de filmes de melhores propriedades, enquanto que sobrepotenciais mais positivos não promovem suficiente formação de hidroxilas. Para sobrepotenciais mais negativos a integridade do revestimento é prejudicada pela evolução de hidrogênio da reação catódica de eletrólise da água, cujas bolhas provocam a ruptura do filme, aumentando a presença de poros.

Considerando-se que a reação relevante para o procedimento, a eletrólise da água, é a mesma para qualquer precursor em sol-gel, é possível que os fenômenos observados se repitam para todos os organossilanos. Deste modo, foram escolhidos os seguintes potenciais para a deposição eletroassistida de VTES, que cobrem as três possibilidades citadas: $-0,4 \mathrm{~V}_{\text {OCP }} ;-0,8 \mathrm{~V}_{\text {OCP }}$ e $-\mathrm{I}, 2 \mathrm{~V}_{\text {OCP }}$

\subsection{Corrosão Acelerada em Névoa Salina}

Os graus de corrosão observados ao longo do ensaio de névoa salina são representados graficamente na Figura 2. Vale destacar que os graus de corrosão não possuem dígito decimal, portanto foi acrescentado a cada série um dígito decimal diferente para evitar a sobreposição de linhas. Deste modo, todos os pontos localizados entre dois graus de corrosão são, na verdade, referentes ao mais baixo destes dois.

A amostra Branco apresentou corrosão antes de todas as amostras pré-tratadas, após 124 dias de ensaio em névoa salina. Após 174 dias, todas as amostras com pré-tratamento de VTES apresentaram corrosão de grau 3 , 


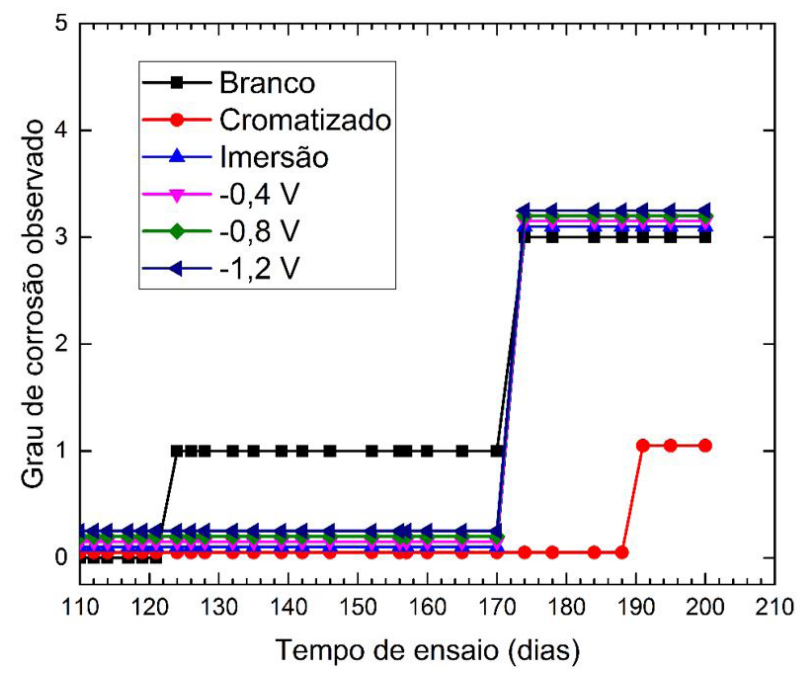

Figura 2. Representação gráfica dos graus de corrosão observados ao longo do ensaio de corrosão acelerada em névoa salina.

situação que durou até o término do ensaio, em 200 dias. O processo de cromatização resultou nos revestimentos mais protetores, apresentando corrosão de grau I apenas após 191 dias de ensaio, possivelmente em função da proteção por barreira fornecida por compostos de cromo trivalente somada à presença de íons cromato solúveis, que atuam na passivação do substrato em áreas afetadas por corrosão [9].

Estes resultados indicam que o VTES é eficiente na proteção contra a corrosão do substrato, tendo retardado o aparecimento de corrosão em 50 dias quando comparado com a amostra sem pré-tratamento, confirmando o caráter protetor do filme de VTES também para o alumínio.

\subsection{Aderência de Revestimento Orgânico}

Os valores de aderência do revestimento orgânico medidos por tração são mostrados na Tabela $I$.

O VTES favoreceu a aderência do revestimento orgânico, proporcionando aumento quando comparado à cromatização e às amostras sem pré-tratamento. Este resultado é condizente com o desempenho observado por Zand e Mahdavian [ 17], quando o viniltrimetoxissilano (VTMS), que difere do VTES apenas pelo tamanho de seus grupos hidrolisáveis, mantendo o mesmo grupamento funcional, promoveu a aderência de revestimento poliuretânico.

Foi também observado um crescimento na aderência do revestimento orgânico em consequência do uso da deposição eletroassistida, com os sobrepotenciais $-0,8 \mathrm{Ve}-1,2 \mathrm{~V}$ resultando nas maiores aderências, superando o pré-tratamento por imersão simples.

Não foi observada uma relação direta entre a aderência do revestimento orgânico e a resistência à corrosão das amostras em ensaio de corrosão acelerada em névoa salina, uma vez que amostras como Imersão e - I,2 V, com diferença significativa de aderência, apresentaram o mesmo desempenho em névoa salina. Já para a amostra Cromatizado, o desempenho superior em ensaio de névoa salina mesmo
Tabela I. Medidas de aderência de revestimento orgânico por tração com equipamento portátil para todos os pré-tratamentos estudados

\begin{tabular}{ccc}
\hline Pré-tratamento & $\begin{array}{c}\text { Aderência média } \\
(\mathbf{M P a})\end{array}$ & $\begin{array}{c}\text { Desvio padrão } \\
(\mathbf{M P a})\end{array}$ \\
\hline Branco & 0,22 & 0,03 \\
Cromatizado & 0,29 & 0,09 \\
Imersão & 0,68 & 0,16 \\
$-0,4 \mathrm{~V}$ & $\mathrm{I}, 08$ & 0,34 \\
$-0,8 \mathrm{~V}$ & $\mathrm{I}, 44$ & 0,36 \\
$-1,2 \mathrm{~V}$ & $\mathrm{I}, 53$ & $0,3 \mathrm{I}$ \\
\hline
\end{tabular}

com baixa aderência de tinta pode ser justificado pelo efeito de passivação pelos cromatos solúveis presentes sob a camada orgânica [2I].

\subsection{MEV e EDS}

Abaixo, na Figura 3, são apresentadas as micrografias por elétrons retroespalhados das amostras com VTES e da amostra não tratada.

As tonalidades observadas em micrografias por elétrons retroespalhados são determinadas pelo número atômico dos elementos presentes, pois o tamanho do núcleo atômico afeta o espalhamento de elétrons. Sendo assim, elementos de maior número atômico apresentam tonalidade mais clara. Os números atômicos do silício e do alumínio são, respectivamente, 14 e 13 , portanto as tonalidades claras dão indício da distribuição do filme na superfície.

Observa-se que tanto a amostra Imersão (Figura 3b) quanto a - $0,4 \mathrm{~V}$ (Figura 3c) apresentam filmes com menor homogeneidade na cobertura do substrato, enquanto que as amostras - 0,8 V (Figura 3d) e - I,2 V (Figura 3e) apresentam maior homogeneidade na cobertura, confirmando as observações de outros autores com relação à maior homogeneidade de filmes produzidos por esta técnica $[1,18,22]$. Nota-se que a amostra - I,2 $\vee$ apresenta mais áreas escuras, indicando a existência de poros no filme de VTES, possivelmente em decorrência da evolução excessiva de hidrogênio na formação do filme.

De modo a complementar a análise por microscopia foram obtidos por EDS mapas da distribuição de silício, cuja presença é representada pelos pontos brancos e em tons de cinza, nestas mesmas áreas. Estas imagens são mostradas na Figura 4.

As imagens da distribuição de silício corroboram as observações feitas a partir das micrografias por elétrons retroespalhados, indicando maior homogeneidade na cobertura fornecida pelos filmes produzidos a $-0,8 \mathrm{~V}$ (Figura 4d) e $-\mathrm{I}, 2 \vee$ (Figura 4e) quando comparados com os demais.

A partir das imagens da Figura 4 foram calculadas as áreas percentuais onde é detectado o silício para cada pré-tratamento. Tais resultados são apresentados na Tabela 2.

As áreas proporcionais apresentadas na Tabela 2 dão mais uma evidência de que a deposição eletroassistida 

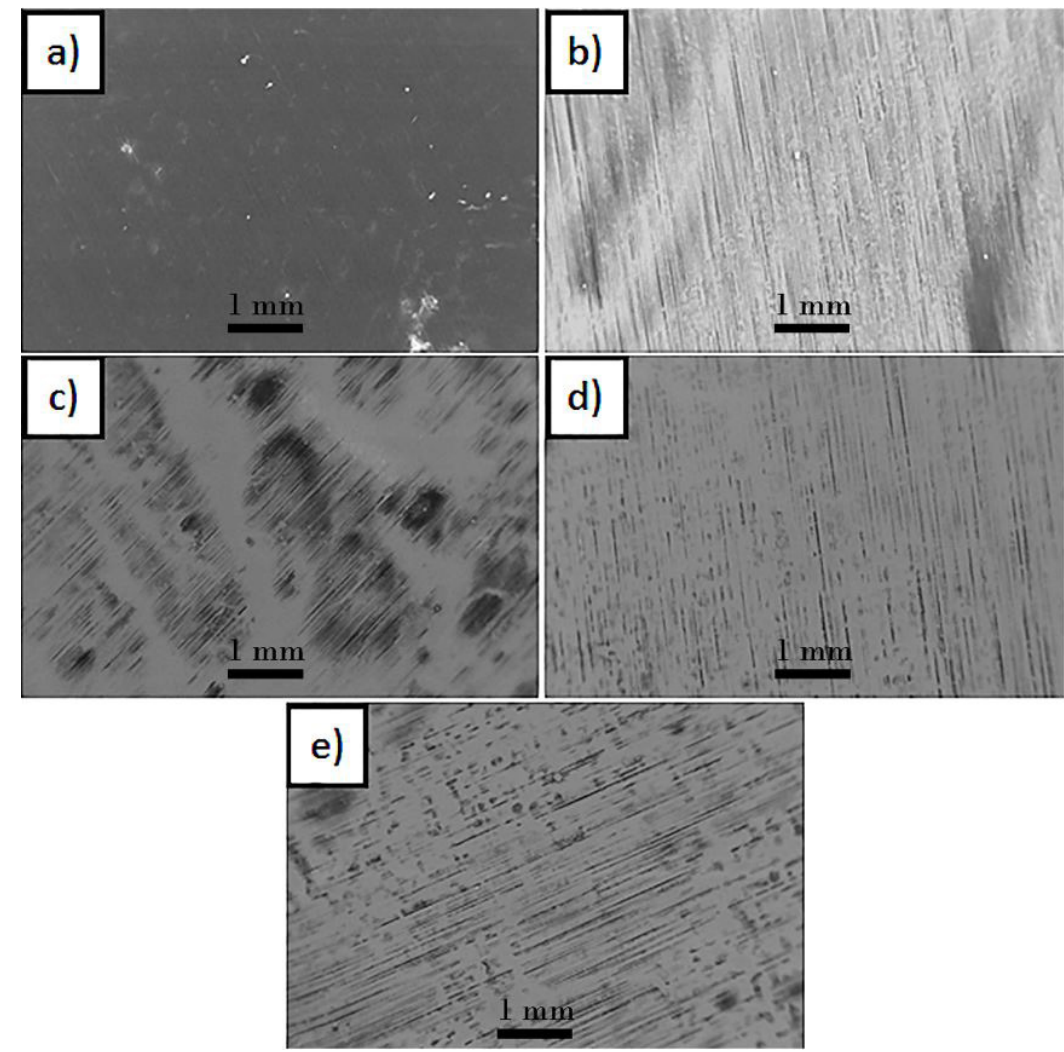

Figura 3. Micrografias por elétrons retroespalhados das amostras (a) Branco, (b) Imersão, (c) - 0,4 V, (d) - 0,8 V e (e) - I, 2 V. Magnificação de 52 vezes.
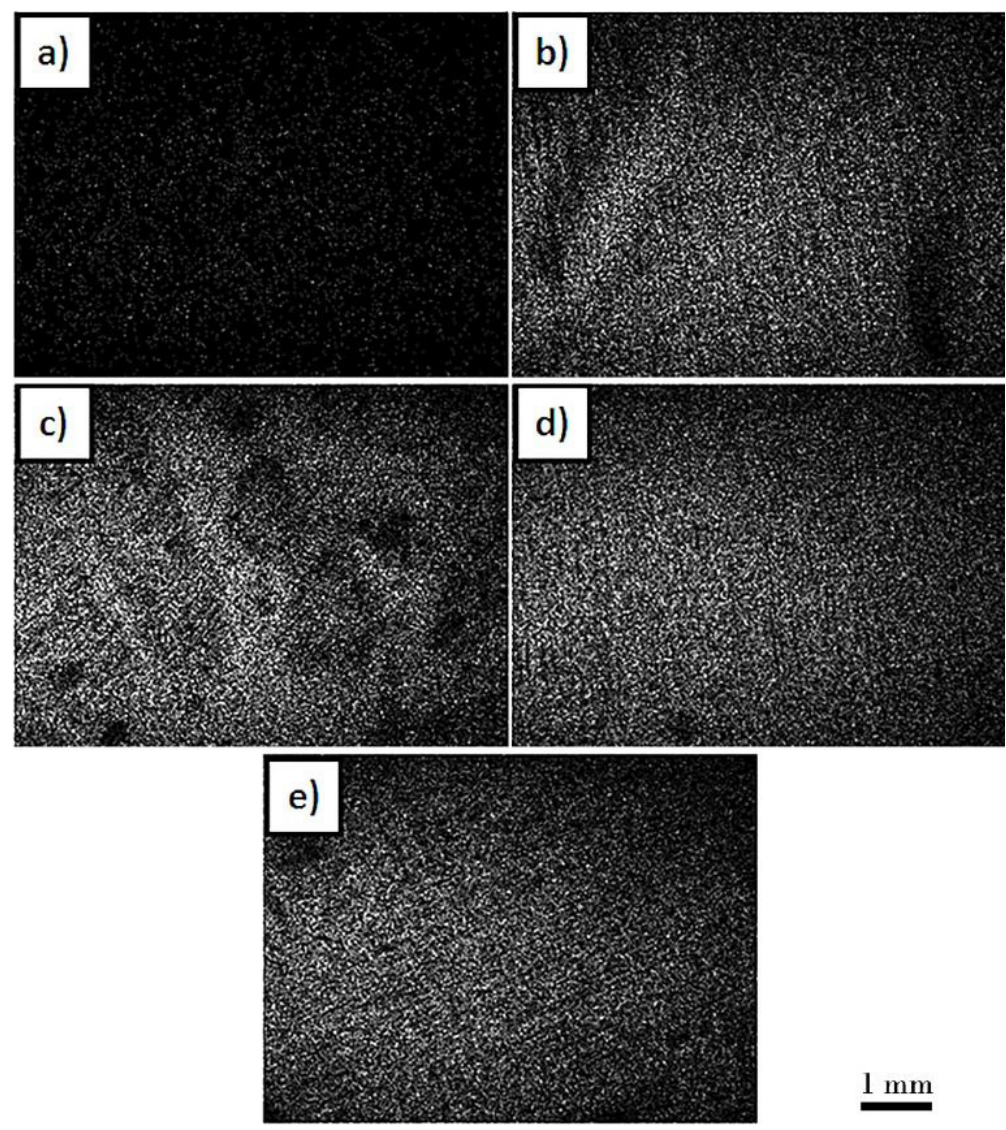

Figura 4. Mapas de silício obtidos por EDS de amostras revestidas com VTES. (a) Branco, (b) Imersão, (c) - 0,4 V, (d) -0,8 V e (e) - I,2 V. 
Tabela 2. Áreas proporcionais contendo silício calculadas a partir dos mapas de silício obtidos por EDS

\begin{tabular}{cc}
\hline Pré-tratamento & $\begin{array}{c}\text { Área proporcional } \\
\text { contendo silício (\%) }\end{array}$ \\
\hline Branco & 2,4 \\
Imersão & 21,6 \\
$-0,4 \mathrm{~V}$ & 23,9 \\
$-0,8 \mathrm{~V}$ & 28,2 \\
$-I, 2 \mathrm{~V}$ & 25,4 \\
\hline
\end{tabular}

com o sobrepotencial $-0,8 V_{\text {ocP }}$ resulta na formação mais bem-sucedida do filme de VTES.

\section{CONCLUSÃO}

O VTES forneceu proteção contra a corrosão ao alumínio, retardando o aparecimento de corrosão em 50 dias durante ensaio de corrosão acelerada em névoa salina. Além dessa proteção, o uso do VTES proporcionou aumento da aderência do revestimento poliuretânico, sendo que a técnica de deposição eletroassistida resultou nos maiores valores de aderência de tinta medidos.

A caracterização morfológica dos filmes de VTES produzidos confirma o êxito no uso da técnica de deposição eletroassistida para a produção de filmes mais homogêneos e com maior capacidade de cobertura, resultando que $o$ sistema de revestimento constituído pelo pré-tratamento de VTES por deposição eletroassistida seguido pela pintura com o revestimento poliuretânico estudado é eficiente para a proteção do alumínio.

\section{Agradecimentos}

Os autores agradecem à equipe do Laboratório de Corrosão, Proteção e Reciclagem de Materiais (LACOR) da Universidade Federal do Rio Grande do Sul e ao Conselho Nacional de Desenvolvimento Científico e Tecnológico (CNPq).

\section{REFERÊNCIAS}

I Gandhi JS. Electrodeposition of organofunctional silanes for improved corrosion protection of metals. . Cincinnati: University of Cincinnati, 2004.

2 Van Ooij WJ, Zhua D, Stacy M, Seth A, Mugada T, Gandhi J, et al. Corrosion protection properties of organofunctional silanes-an overview. Tsinghua Science and Technology. 2005; 10(6):639-664.

3 Ciobotaru I-A, Maior I, Vaireanu D-I, Cojocaru A, Caprarescu S, Ciobotaru I-E. The determination of the optimum hydrolysis time for silane films deposition. Applied Surface Science. 2016;371:275-280.

4 Shacham R, Avnir D, Mandler D. Electrodeposition of methylated sol-gel films on conducting surfaces. Advanced Materials. 1999; I I(5):384-388.

5 Cao Z, Wang H, Qu J, Zhang M, Wang X, Xia W. One step GO/DTES co-deposition on steels: Electro-induced fabrication and characterization of thickness-controlled coatings. Chemical Engineering Journal. 2017;320:588-607.

6 Liu Y-H, Xu J-B, Zhang J-T, Hu J-M. Electrodeposited silica film interlayer for active corrosion protection. Corrosion Science. 2017

7 Joseph R. ABCs of painting on aluminum. Metal Finishing. 2008;6(2):39-44.

8 Chen P-N. The effect of chromate on adhesion between epoxy coating and Al substrate. Bethlehem: Lehigh University; 1999.

9 Schweitzer PA. Corrosion engineering handbook. cathodic and inhibitor protection and corrosion monitoring: corrosion of linings and coatings. 2nd ed. Boca Raton: CRC Press; 2007.

10 Kalidhasan S, Santhana Krishna Kumar A, Rajesh V, Rajesh N. The journey traversed in the remediation of hexavalent chromium and the road ahead toward greener alternatives-a perspective. Coordination Chemistry Reviews. 2016;317:157-166.

I I Zheludkevich ML, Salvado IM, Ferreira MGS. Sol-gel coatings for corrosion protection of metals. Journal of Materials Chemistry. 2005; I5(48):5099.

12 Yuan X, Yue ZF, Chen X, Wen SF, Li L, Feng T. The protective and adhesion properties of silicone-epoxy hybrid coatings on 2024 Al-alloy with a silane film as pretreatment. Corrosion Science. 2016;104:84-97.

13 Ramezanzadeh B, Raeisi E, Mahdavian M. Studying various mixtures of 3-aminopropyltriethoxysilane (APS) and tetraethylorthosilicate (TEOS) silanes on the corrosion resistance of mild steel and adhesion properties of epoxy coating. International Journal of Adhesion and Adhesives. 2015;63:166-I76.

14 Wang J, Wu L-K, Zhou J-H, Hu J-M, Zhang J-Q, Cao C-N. Construction of a novel painting system using electrodeposited $\mathrm{SiO} 2$ film as the pretreatment layer. Corrosion Science. 20I3;68:57-65. 
I5 Liu Y-H, Jin X-H, Hu J-M. Electrodeposited silica films post-treated with organosilane coupling agent as the pretreatment layers of organic coating system. Corrosion Science. 2016; 106:127-1 36.

16 Sacilotto DG, Ferreira JZ. Influência da modificação superficial sobre a resistência à corrosão do aço inoxidável AISI 204 com revestimento hidrofóbico. Tecnologica em Metalurgia, Materiais e Mineração. 2016;13(2):20I-208.

17 Zand BN, Mahdavian M. Corrosion and adhesion study of polyurethane coating on silane pretreated aluminum. Surface and Coatings Technology. 2009;203(12):1677-168I.

$18 \mathrm{Hu}$ J-M, Liu L, Zhang J-Q, Cao C-N. Effects of electrodeposition potential on the corrosion properties of bis-1,2[triethoxysilyl] ethane films on aluminum alloy. Electrochimica Acta. 2006;5 I (19):3944-3949.

$19 \mathrm{Hu}$ J-M, Liu L, Zhang J-Q, Cao C-N. Electrodeposition of silane films on aluminum alloys for corrosion protection. Progress in Organic Coatings. 2007;58(4):265-27I.

20 Li M, Yang Y-Q, Liu L, Hu J-M, Zhang J-Q. Electro-assisted preparation of dodecyltrimethoxysilane/TiO2 composite films for corrosion protection of AA2024-T3 (aluminum alloy). Electrochimica Acta. 2010;55(8):3008-30I4.

2I Zhao J, Xia L, Sehgal A, Lu D, McCreery RL, Frankel GS. Effects of chromate and chromate conversion coatings on corrosion of aluminum alloy 2024-T3. Surface and Coatings Technology. 200 I; I40(I):5I-57.

22 Chandrasekaran S. Electrodeposition of bis-silane for the pretreatment of aluminum alloys. Cincinnati: University of Cincinnati; 2006.

Recebido em: 16 Jan. 2017

Aceito em: 22 Maio 2017 